\title{
ASYMPTOTIC SOLUTIONS OF A GENERALIZED BURGERS EQUATION
}

\author{
BY \\ V. VANAJA AND P. L. SACHDEV * \\ New Jersey Institute of Technology, Newark, New Jersey
}

Abstract. The travelling wave solutions of the generalized Burgers equation

$$
\frac{\partial u}{\partial t}=\frac{\partial}{\partial x}\left[D(u) \frac{\partial u}{\partial x}\right]-\frac{\partial}{\partial x}[K(u)]
$$

are related to the solution of the initial boundary value problems for the same equation, subject to initial boundary conditions relevant to the physical problem of infiltration of moisture into a homogeneous soil. The theoretical prediction of the emergence of the travelling wave solutions as intermediate asymptotics is confirmed by numerical solutions of the problem for some specific choices of the functions $D(u)$ and $K(u)$.

1. Introduction. In this paper, we study intermediate asymptotic solutions to the problem (1.1), (1.2). Let the domain $E$ be given by $[(x, t): 0<x<\infty, 0<t<$ $\infty]$.

$$
\frac{\partial u}{\partial t}=\frac{\partial}{\partial x}\left[D(u) \frac{\partial u}{\partial x}\right]-\frac{\partial}{\partial x}[K(u)] \text { for }(x, t) \text { in } E .
$$

Let $D(u), D^{\prime}(u), K(u), K^{\prime}(u), K^{\prime \prime}(u)>0$ exist, be continuous and bounded when $u \geq u_{0}>0$, where $u_{0}$ is a constant.

$$
\begin{array}{ll}
u(0, t)=u_{1} & \text { (constant) for } 0 \leq t<\infty, \\
u(x, 0)=u_{0}(x) & \text { for } 0 \leq x<\infty \\
u_{0} \leq u_{0}(x) \leq u_{1}, & \lim _{x \rightarrow \infty} u_{0}(x)=u_{0} .
\end{array}
$$

We first describe the physical situation giving rise to this problem. It is described in detail in [2] and [12]. For further references, the reader may refer to $[3,8,16,6]$. Equation (1.1) describes the flow of water in a porous medium [2, 12]. We consider the one-dimensional flow of water under gravity through a homogeneous isotropic porous medium. We define the local quantity $u$ as the volume of water per unit volume of porous medium and $q$ as the volume of water flowing across unit area per

Received September 19, 1990.

${ }^{*}$ On leave from the Department of Mathematics, Indian Institute of Science, Bangalore, India.

(C) 1992 Brown University 
unit time. If the density of water is assumed constant, then the motion is governed by the continuity equation

$$
\frac{\partial u}{\partial t}-\frac{\partial q}{\partial x}=0
$$

and Darcy's law

$$
q=K(u) \frac{\partial G}{\partial x},
$$

where $x$ is the space coordinate measured positive downward, $t$ is the time, and $K(u)$ is the hydraulic conductivity. Under certain conditions for unsaturated flows the potential $G$ may be expressed as the sum of a gravitational potential and a capillary potential $H(u)$ due to capillary suction. Thus we put

$$
G=H(u)-x
$$

and Eqs. (1.3), (1.4) become

$$
\frac{\partial u}{\partial t}=\frac{\partial}{\partial x}\left[K(u) \frac{\partial H}{\partial x}\right]-\frac{\partial}{\partial x}[K(u)] .
$$

If we let

$$
D(u)=K(u) \frac{\partial H}{\partial u}
$$

in Eq.(1.6), we get Eq.(1.1). Empirical analytic expressions for $D(u)$ and $K(u)$ of a power-law type are known $[2,12]$ to be

$$
D(u)=D_{0} u^{m-1}, \quad K(u)=K_{0} u^{n},
$$

where $D_{0}, K_{0}, m$, and $n$ are positive constants and $n \geq m>1$. Hence rescaling the independent variables we finally have

$$
\frac{\partial u}{\partial t}=\frac{\partial^{2}\left(u^{m}\right)}{\partial x^{2}}-\frac{\partial\left(u^{n}\right)}{\partial x}, \quad \text { where } n \geq m>1 .
$$

So, during infiltration of water into a homogeneous soil, the moisture $u(x, t)$ of the soil satisfies an equation of the form (1.1). Taking into account the initial moisture distribution in the soil and infiltration on the surface of the ground, we obtain the boundary conditions for $x=0$ and for $t=0$ stated in (1.2). The boundary condition at $x=0$, namely $u_{1}$, is used with the understanding that $u_{1}=1$ denotes the moisture corresponding to full saturation of the soil on the surface of the ground. We also assume that there is no water at a large depth beneath the ground.

The role of self-similar or travelling wave solutions in the asymptotic behaviour of initial/boundary value problems of partial differential equations is now well established [13]. These are often called intermediate asymptotics [1]. One of the earlier studies in this context is due to Serrin [15]. Peletier and his co-investigators have studied several nonlinear parabolic equations [17, 18], including the nonlinear heat equation [11]. Fisher's equation and its generalizations have been treated by Canosa $[4,5]$ (see also [13]).

Nonlinear parabolic equations with convective and geometric terms-generalized Burgers equations (GBE) - have been studied by Scott [14]. He has shown that the 
solutions of initial value problems

$$
\begin{gathered}
u_{x}-u u_{t}=g(x) u_{t t} \quad \text { for } 0 \leq x<\infty, \\
g(x) / x \rightarrow \beta \neq 0 \text { as } x \rightarrow \infty, \\
u(0, t)=u_{0}(t)
\end{gathered}
$$

tend asymptotically to the similarity solution $u=\Omega(t / x)$ of the equation

$$
u_{x}-u u_{t}=\beta x u_{t t}
$$

as $x \rightarrow \infty$. In the present paper, we determine the travelling wave solution to the problem (1.1), (1.2), the former being another GBE.

In [9], an attempt was made to get an integral estimate for the difference between the solution of the problem and its travelling wave solution and hence a pointwise estimate for the same. The details in this work are not clear. In the present paper, we obtain a pointwise estimate of the difference of the solution of the initial/boundary value problem (1.1), (1.2) and its travelling wave solution directly. The method of proof for the main theorem in this paper is similar to that in [11].

This paper is organized as follows. In Sec. 2, we prove the existence of the travelling wave solution to the problem (1.1), (1.2) and some of its properties. In Sec. 3 , we prove the main result concerning the solution of the problem and its travelling wave solution. In Sec. 4, we present some numerical results for the problem (1.7), (1.2), which is a special case of (1.1), (1.2). In Sec. 5, we make some concluding remarks.

2. Travelling wave solution and some of its properties. We first consider travelling wave solutions for the problem (1.1), (1.2). Writing $\eta=x-A t+c$, where $A$ is a positive constant, Eq. (1.1) reduces to the second-order ordinary differential equation

$$
\frac{d}{d \eta}\left[D(\mathscr{U}) \frac{d \mathscr{U}}{d \eta}\right]-\frac{d}{d \eta}[K(\mathscr{U})]=-A \mathscr{U}_{\eta} .
$$

We impose the end conditions

$$
\mathscr{U}(-\infty)=u_{1}, \quad \mathscr{U}(+\infty)=u_{0} .
$$

We first prove the existence of the travelling wave solution in

THEOREM 2.1. If $u_{0}<u_{2}<u_{1}$, where $\mathscr{U}(0)=u_{2}$, then a travelling wave solution $\mathscr{U}(x-A t+c)$ of $(1.1),(1.2)$ exists.

Proof. Integrating (2.1) once with respect to $\eta$, and imposing the condition at $+\infty$ yields

$$
D(\mathscr{U}) \frac{d \mathscr{U}}{d \eta}-K(\mathscr{U})+K\left(u_{0}\right)=-A\left(\mathscr{U}-u_{0}\right) .
$$

Imposing the condition at $-\infty$ yields

$$
A=\left(K\left(u_{1}\right)-K\left(u_{0}\right)\right) /\left(u_{1}-u_{0}\right) .
$$

Integrating (2.3) again gives

$$
x-A t+c=\int_{u_{2}}^{\mathscr{U}} \frac{D(u)}{\left[K^{\prime}\left(u_{0}+\theta\left(u-u_{0}\right)\right)-A\right]\left[u-u_{0}\right]} d u,
$$

where $0<\theta(u)<1$. This finishes the proof of Theorem 2.1. 
COROLlary 2.1. The travelling wave solution is a monotonic decreasing function of $\eta$.

Proof. By differentiating both sides of (2.5) with respect to $x$, we obtain, after some simplification,

$$
\frac{d \mathscr{U}}{d \eta}=\frac{\left[K^{\prime}\left(u_{0}+\theta\left(\mathscr{U}-u_{0}\right)\right)-A\right]\left(\mathscr{U}-u_{0}\right)}{D(\mathscr{U})} .
$$

If we expand $K(\mathscr{U})$ in a Taylor series about $u_{0}$, we obtain $K^{\prime}\left(u_{0}+\theta\left(\mathscr{U}-u_{0}\right)\right) \leq A$. Since $D(\mathscr{U}), \mathscr{U}-u_{0} \geq 0$, the result follows.

For the problem (1.7), (1.2), similar results are true. We simply state the formula for the travelling wave solution in the following remark without proving it.

REMARK 2.1. If $u_{0}<u_{2}<u_{1}$, then a travelling wave solution $\mathscr{U}(x-A t+c)$ $(A>0)$ of (1.7), (1.2) exists and is given by the following:

$$
(x-A t+c)=\int_{u_{2}}^{\mathscr{U}} \frac{m u^{m-1}\left[u_{1}-u_{0}\right]}{\left[\left(u_{1}-u_{0}\right)\left(u^{n}-u_{0}^{n}\right)-\left(u-u_{0}\right)\left(u_{1}^{n}-u_{0}^{n}\right)\right]} d u .
$$

We denote by $E^{+}$the domain bounded by $x=0, t=0$, and $t=T$, where $T$ is any finite positive number.

We next state a minimum principle, which is used several times in this paper. It is analogous to the maximum principle due to Krzyzanski [10].

LEMMA 2.1. Let $z(x, t)$ be a bounded solution of the differential inequality

$$
a(x, t) z_{x x}+b(x, t) z_{x}+c(x, t) z-z_{t} \leq 0
$$

in $E^{+}$. Let $a(x, t), b(x, t)$, and $c(x, t)$ be bounded continuous functions of $x$ and $t$ and $a(x, t)>0$. Then, if $z \geq 0$ on $x=0$ and $t=0$, then $z \geq 0$ in $E^{+}$.

We next prove the following theorem, which is then used to prove Theorem 2.3.

THEOREM 2.2. If $u, v$ are two solutions of $(1.1),(1.2)$ in $E$, and $u \leq v$ on $x=0$ and $t=0$, then $u \leq v$ in $E$.

Proof. We set $\bar{u}=\int_{0}^{u} D(s) d s$ and $\bar{v}=\int_{0}^{\prime \prime} D(s) d s$. Then $\bar{u}$ and $\bar{v}$ satisfy

$$
\bar{u}_{t}=D(u) \bar{u}_{x x}-K^{\prime}(u) \bar{u}_{x}, \quad \bar{v}_{t}=D(v) \bar{v}_{x x}-K^{\prime}(v) \bar{v}_{x} .
$$

If $w=\bar{v}-\bar{u}$, then $w$ satisfies

$$
w_{t}=D(v) w_{x x}-K^{\prime}(v) w_{x}+\bar{u}_{x x}[D(v)-D(u)]-\bar{u}_{x}\left[K^{\prime}(v)-K^{\prime}(u)\right] .
$$

But, $D(v)-D(u)=(v-u) D^{\prime}\left(\theta_{1}\right)$ and $K^{\prime}(v)-K^{\prime}(u)=(v-u) K^{\prime \prime}\left(\theta_{2}\right)$, where $\theta_{1}=\theta_{1}(x, t)$ and $\theta_{2}=\theta_{2}(x, t)$ lie between $u$ and $v$. Also, $\bar{v}-\bar{u}=(v-u) D\left(\theta_{3}\right)$ for some $\theta_{3}=\theta_{3}(x, t)$ between $u$ and $v$. So, Eq. (2.6) becomes

$$
\begin{aligned}
w_{t}= & D(v) w_{x x}-K^{\prime}(v) w_{x} \\
& +w\left[\left(\bar{u}_{x x}(v-u) D^{\prime}\left(\theta_{1}\right)\right) /(\bar{v}-\bar{u})-\left(\bar{u}_{x}(v-u) K^{\prime \prime}\left(\theta_{2}\right)\right) /(\bar{v}-\bar{u})\right] .
\end{aligned}
$$

Therefore we get

$$
D(v) w_{x x}-K^{\prime}(v) w_{x}+\xi(x, t) w-w_{t}=0,
$$


where

$$
\xi(x, t)=\left[\left(\bar{u}_{x x} D^{\prime}\left(\theta_{1}\right)\right) /\left(D\left(\theta_{3}\right)\right)-\left(\bar{u}_{x} K^{\prime \prime}\left(\theta_{2}\right)\right) /\left(D\left(\theta_{3}\right)\right)\right] .
$$

Here, $D, K^{\prime}$, and $\xi(x, t)$ are bounded in $E$. Since $D>0$ and $w=$ $(v-u) D\left(\theta_{3}\right) \geq 0$ on $x=0$ and $t=0, w$ satisfies the conditions of Lemma 2.1. It follows that $w \geq 0$ in $E^{+}$. But the proof is independent of the choice of $T$. Hence the result is true for all $t \geq 0$.

Corollary 2.2. If $u(x, t)$ is the solution of the problem (1.1), (1.2), then $u_{0} \leq$ $u(x, t) \leq u_{1}$ for each $(x, t)$ in $E$.

Proof. Applying Theorem 2.2 to $u(x, t)$ and $u_{1}$, we get the inequality $u(x, t) \leq$ $u_{1}$. Similarly, the other inequality can be proved.

Let us assume that the initial moisture distribution $u_{0}(x)$ satisfies

$$
u_{0}(x)-u_{0} \leq M_{1} e^{-\gamma_{1} x}
$$

where $\gamma_{1}>\left|K^{\prime}\left(u_{0}\right)-A\right| / D\left(u_{0}\right)$ is a positive constant and $M_{1}$ is a constant $>0$. A bound for $M_{1}$ is specified later in (2.13). In the next theorem, we prove that the solution of the problem (1.1), (1.2) can be bounded by two specific travelling wave solutions.

THEOREM 2.3. Let $u(x, t)$ be the solution of the problem (1.1), (1.2) and let $u_{0}(x)$ satisfy condition (2.8). Then there exist travelling wave solutions $\mathscr{U}_{1}\left(x-\lambda_{1} t+c_{1}\right)$ and $\mathscr{U}_{2}\left(x-\lambda_{2} t-c_{2}\right)$, where $\lambda_{1}, \lambda_{2}, c_{1}, c_{2}>0$, and $\mathscr{U}_{1}(-\infty)=u_{1}, \mathscr{U}_{1}(+\infty)=u_{0}-\varepsilon$ $(\varepsilon>0), \mathscr{U}_{2}(-\infty)=u_{1}+\varepsilon$, and $\mathscr{U}_{2}(+\infty)=u_{0}$, so that the following is true:

$$
\mathscr{U}_{1}\left(x-\lambda_{1} t+c_{1}\right) \leq u(x, t) \leq \mathscr{U}_{2}\left(x-\lambda_{2} t-c_{2}\right) \text { for every }(x, t) \text { in } E .
$$

Proof. Notice that $\lambda_{2}=\left[\left(K\left(u_{1}+\varepsilon\right)-K\left(u_{0}\right)\right) /\left(u_{1}+\varepsilon-u_{0}\right)\right]>A$. We let $m_{2}=$ $\left|K^{\prime}\left(u_{0}\right)-\lambda_{2}\right| / D\left(u_{0}\right)$. We then choose $\varepsilon>0$ sufficiently small so that $\gamma_{1} \geq m_{2}>$ $\left|K^{\prime}\left(u_{0}\right)-A\right| / D\left(u_{0}\right)$. This is possible due to the choice of $\gamma_{1}$ given by the second inequality in $(2.8)$.

Since the travelling wave $\mathscr{U}$ and $u_{0}(x)$ are both decreasing, there exist travelling wave solutions of the form $\mathscr{U}_{2}, \mathscr{U}_{1}$ satisfying the end conditions mentioned in the statement of the theorem, so that

$$
\begin{array}{ll}
u_{0}(x) \leq \mathscr{U}_{2}\left(x-c_{2}\right) & \text { for each } x>0, \\
u_{0}(x) \geq \mathscr{U}_{1}\left(x+c_{1}\right) & \text { for each } x>0 .
\end{array}
$$

Also, on $x=0$ we have $\mathscr{U}_{1} \leq u_{1} \leq \mathscr{U}_{2}$. So, by Theorem 2.2 , the result follows.

Corollary 2.3. $\lim _{x \rightarrow \infty} u(x, t)=u_{0}$ for any finite $t$.

Proof. Since both $\mathscr{U}_{1}^{\infty}\left(x-\lambda_{1} t+c_{1}\right)$ and $\mathscr{U}_{2}\left(x-\lambda_{t} t-c_{2}\right)$ in (2.9) tend to $u_{0}$ in the limit, the result follows.

REMARK 2.2. It is proved in [9] that

$$
\mathscr{U}_{2}-u_{0} \leq e^{c_{2} m_{2}} e^{-m_{2}\left(x-\lambda_{2} t\right)} .
$$

From (2.8), (2.9), and (2.12), we get

$$
M_{1} \leq e^{c_{2} m_{2}} .
$$


It is also proved in [9] that for suitably chosen $\lambda_{1}$

$$
u_{1}-\mathscr{U}_{1} \leq k_{1} e^{m_{1}\left(x-\lambda_{1} t\right)},
$$

where $m_{1}=\left|K^{\prime}\left(u_{1}\right)-\lambda_{1}\right| / D\left(u_{0}\right)$ and $k_{1}$ is a positive constant.

3. Main result. In this section, we prove the main result regarding how close the solutions of the problem (1.1), (1.2) get to its travelling wave solution.

THEOREM 3.1. Let $u(x, t)$ be the solution of $(1.1),(1.2)$ and $\mathscr{U}(x-A t+c)$ be the travelling wave solution. Let the initial profile $u_{0}(x)$ satisfy condition (2.8). Then there exist constants $M, l>0$, and $c$ so that the following is true:

$$
|u(x, t)-\mathscr{U}(x-A t+c)| \leq M e^{-l t} \text { for each }(x, t) \text { in } E .
$$

Proof. Let us choose the constant $c$ such that $K\left(u_{0}\right)-A u_{0}+c=K\left(u_{1}\right)-A u_{1}+c=$ 0 . As in the proof of Theorem 2.2, we set $\bar{u}=\int_{0}^{u} D(s) d s, \overline{\mathscr{U}}=\int_{0}^{\mathscr{U}} D(s) d s$, and $y=\bar{u}-\overline{\mathscr{U}}$ to obtain

$$
\mathscr{L}(y)=D(u) y_{x x}-K^{\prime}(u) y_{x}+\beta(x, t) y-y_{t}=0,
$$

where

$$
\beta(x, t)=\left[\left(\overline{\mathscr{U}}_{x x} D^{\prime}\left(\theta_{4}\right)\right) /\left(D\left(\theta_{6}\right)\right)-\left(\overline{\mathscr{U}}_{x} K^{\prime \prime}\left(\theta_{5}\right)\right) /\left(D\left(\theta_{6}\right)\right)\right] ;
$$

here $\theta_{4}=\theta_{4}(x, t), \theta_{5}=\theta_{5}(x, t)$, and $\theta_{6}=\theta_{6}(x, t)$ all lie between $u$ and $\mathscr{U}$. We claim that $\beta(x, t)$ is bounded. We write $\mathscr{U}_{\eta}$ for $\frac{d \mathscr{U}}{d \eta}$. Recalling that $\eta=x-A t+c$, since $\overline{\mathscr{U}}_{x}=D(\mathscr{U}) \mathscr{U}_{\eta}$, we get

$$
\overline{\mathscr{U}}_{x x}=\frac{d}{d \eta}\left[D(\mathscr{U}) \mathscr{U}_{\eta}\right]=\frac{d}{d \eta}[K(\mathscr{U})]-A \mathscr{U}_{\eta}=\left[K^{\prime}(\mathscr{U})-A\right] \mathscr{U}_{\eta},
$$

where we have used (2.3). Hence, we get

$$
\beta(x, t)=\left(-\mathscr{U}_{\eta}\right)\left[\left(A-K^{\prime}(\mathscr{U})\right) D^{\prime}\left(\theta_{4}\right) / D\left(\theta_{6}\right)+D(\mathscr{U}) K^{\prime \prime}\left(\theta_{5}\right) / D\left(\theta_{6}\right)\right] .
$$

Since $\mathscr{U}_{\eta}=\left[K^{\prime}\left(u_{0}+\theta\left(\mathscr{U}-u_{0}\right)\right)-A\right]\left(\mathscr{U}-u_{0}\right) / D(\mathscr{U})$, where $K^{\prime}\left(u_{0}+\theta\left(\mathscr{U}-u_{0}\right)\right) \leq A$, $\mathscr{U} \leq u_{1}$, and $D(\mathscr{U}) \geq D\left(u_{0}\right)$ for all $\mathscr{U}$, we get $\left|\mathscr{U}_{\eta}\right| \leq 2 A\left(u_{1}-u_{0}\right) / D\left(u_{0}\right)$ and therefore

$$
|\beta| \leq\left[2 A\left(u_{1}-u_{0}\right) / D\left(u_{0}\right)\right]\left[\left(2 A\left[D^{\prime}\right]+D\left(u_{1}\right)\left[K^{\prime \prime}\right]\right) / D\left(u_{0}\right)\right],
$$

which proves the claim.

Next, we consider the comparison function

$$
z(x, t)=e^{-l t} \omega(\eta),
$$

where $\omega(\eta)$ is a positive, continuous, and piecewise differentiable function which we shall specify shortly. Just as in [11], the function $\omega(\eta)$ has to be chosen carefully in order to make $\mathscr{L}(z) \leq 0$ for all $\eta$. We find that

$$
\mathscr{L}(z)=z\left[D(u) \omega^{\prime \prime} / \omega+\left(A-K^{\prime}(u)\right) \omega^{\prime} / \omega+\beta(x, t)+l\right] .
$$

Now, for $\omega(\eta)$ we choose a function used in [9]:

$$
\omega(\eta)= \begin{cases}e^{-\alpha \exp (\lambda \eta)} & \text { for }|\eta|<N, \\ e^{-\alpha \eta} & \text { for }|\eta| \geq N,\end{cases}
$$


where $\alpha=(1 / k) \exp (-\lambda N), k \geq 1 \quad(\lambda$ and $N$ will be specified later $)$.

$$
\begin{gathered}
\omega^{\prime} / \omega= \begin{cases}-\alpha \lambda e^{\lambda \eta} & \text { for }|\eta|<N, \\
-\alpha & \text { for }|\eta| \geq N .\end{cases} \\
\omega^{\prime \prime} / \omega= \begin{cases}\alpha^{2} \lambda^{2} e^{2 \lambda \eta}-\alpha \lambda^{2} e^{\lambda \eta} & \text { for }|\eta|<N, \\
\alpha^{2} & \text { for }|\eta| \geq N .\end{cases}
\end{gathered}
$$

First, we prove that

$$
\mathscr{L}(z) \leq 0 \text { for all } \eta
$$

Recall that

$$
\mathscr{U}_{\eta}=\left[K^{\prime}\left(u_{0}+\theta\left(\mathscr{U}-u_{0}\right)\right)-A\right]\left(\mathscr{U}-u_{0}\right) / D(\mathscr{U}),
$$

$\mathscr{U} \rightarrow u_{0}$ as $\eta \rightarrow \infty$, and $\left[K^{\prime}\left(u_{0}+\theta\left(\mathscr{U}-u_{0}\right)\right)-A\right] \rightarrow 0$ as $\eta \rightarrow-\infty$. So, we choose $N$ so large that for $|\eta| \geq N, \beta$ given by (3.3) is very small.

Let us first consider the case $|\eta|<N$ :

$$
\begin{aligned}
D(u) \omega^{\prime \prime} / \omega+\left(A-K^{\prime}(u)\right) \omega^{\prime} / \omega+\beta+l & \\
= & D(u) \lambda^{2}\left[1 /\left(k^{2} e^{2 \lambda(N-\eta)}\right)-1 /\left(k e^{\lambda(N-\eta)}\right)\right] \\
& -\left[A-K^{\prime}(u)\right] \lambda /\left(k e^{\lambda(N-\eta)}\right)+\beta+l .
\end{aligned}
$$

Since $D(u) \geq D\left(u_{0}\right)$ and $K^{\prime}(u) \leq K^{\prime}\left(u_{1}\right)$, we choose $\lambda$ sufficiently large that

$$
\begin{aligned}
l_{1}= & D\left(u_{0}\right) \lambda^{2}\left[1 /\left(k e^{\lambda(N-\eta)}\right)-1 /\left(k^{2} e^{2 \lambda(N-\eta)}\right)\right] \\
& +\left[A-K^{\prime}\left(u_{1}\right)\right] \lambda /\left(k e^{\lambda(N-\eta)}\right)-\beta \geq 0 .
\end{aligned}
$$

This is possible since $\beta$ is bounded. Now, we choose $l$ so that $l \leq l_{1}$. For this choice of $l$, we get

$$
\mathscr{L}(z) \leq 0 \text { for }|\eta|<N .
$$

Next, we consider the case $|\eta| \geq N$ :

$$
\begin{aligned}
& D(u) \omega^{\prime \prime} / \omega+\left(A-K^{\prime}(u)\right) \omega^{\prime} / \omega+\beta+l \\
& \quad=D(u) /\left(k^{2} e^{2 \lambda N}\right)-\left(A-K^{\prime}(u)\right) /\left(k e^{\lambda N}\right)+\beta+l .
\end{aligned}
$$

Since $K^{\prime}(u) \leq A$, we let $s=\sup \left[K^{\prime}(u)\right]$. Recalling that $\beta$ can be made arbitrarily small for $|\eta| \geq N$ and since $D(u) \leq D\left(u_{1}\right)$, we choose $k$ sufficiently large that

$$
1 /\left(k^{2} e^{2 \lambda N}\right)+\beta / D\left(u_{1}\right) \leq|A-s| /\left[D\left(u_{1}\right) k e^{\lambda N}\right] .
$$

This would ensure that

$$
l_{2}=\left(\left[(A-s) k e^{\lambda N}-D\left(u_{1}\right)\right] /\left(k^{2} e^{2 \lambda N}\right)\right)-\beta \geq 0 .
$$

Notice that it follows from inequalities (3.6) and (2.8) that

$$
1 /\left(k e^{\lambda N}\right)<|A-s| / D\left(u_{1}\right) \leq\left|A-K^{\prime}\left(u_{0}\right)\right| / D\left(u_{0}\right)<\gamma_{1} .
$$

Now, choose $l$ so that $l \leq l_{2}$. For this choice of $l$,

$$
\mathscr{L}(z) \leq 0 \text { for }|\eta| \geq N \text {. }
$$


Finally, we let $l=\min \left(l_{1}, l_{2}\right)$. This will make $\mathscr{L}(z) \leq 0$ for all $\eta$ and proves the relation (3.5). Let

$$
\phi(x, t)=N_{1} z(x, t)-y(x, t),
$$

where $N_{1}$ is a constant to be chosen. Now, $\phi(0, t)=N_{1} z(0, t)-y(0, t)$. Here, $z(0, t) \geq 0$ and $y(0, t)=\int_{\mathscr{U}(0, t)}^{u_{1}} D(s) d s \geq 0$ because $\mathscr{U}(0, t) \leq u_{1}$. Also, $\phi(x, 0)$ $=N_{1} z(x, 0)-y(x, 0)$, where $z(x, 0)>0$. So, we choose $N_{1}$ sufficiently large that $\phi(0, t)$ and $\phi(x, 0)$ are both $\geq 0$. Now,

$$
\mathscr{L}(\phi)=N_{1} \mathscr{L}(z)-\mathscr{L}(y)=N_{1} \mathscr{L}(z) \leq 0 \text { for all } \eta,
$$

where we have used (3.5) and the fact that $\mathscr{L}(y)=0$. So, by Lemma 2.1 , we get $\phi \geq 0$ everywhere in $E^{+}$. Hence, we get

$$
y(x, t) \leq N_{1} z(x, t) \text { in } E^{+} .
$$

Similarly, if we work with $\psi=N_{2} z+y$, where $N_{2}$ is a constant, then we get

$$
y(x, t) \geq-N_{2} z(x, t) \text { in } E^{+} .
$$

From (3.9) and (3.10) we get

$$
-N_{2} z(x, t) \leq y(x, t) \leq N_{1} z(x, t) \text { in } E^{+} .
$$

So we get

$$
|y|=|\bar{u}-\overline{\mathscr{U}}| \leq|z| \max \left(N_{1}, N_{2}\right)=\left[|\omega(\eta)| /\left(e^{l t}\right)\right] \max \left(N_{1}, N_{2}\right) .
$$

But, $\bar{u}-\overline{\mathscr{U}}=(u-\mathscr{U}) D(\theta(x, t))$, where $\theta$ lies between $u$ and $\mathscr{U}$ and $D(\theta) \geq$ $D\left(u_{0}\right)$. Hence we get

$$
\begin{aligned}
|u-\mathscr{U}| & =|\bar{u}-\overline{\mathscr{U}}| /|D(\theta)| \leq\left[|\omega(\eta)| \max \left(N_{1}, N_{2}\right) / D\left(u_{0}\right)\right] e^{-(l t)} \\
& \leq\left[\max \left(N_{1}, N_{2}\right) / D\left(u_{0}\right)\right] e^{-(l t)},
\end{aligned}
$$

where we have used the fact $|\omega(\eta)| \leq 1$. So, if we set $M=\max \left(N_{1}, N_{2}\right) / D\left(u_{0}\right)$, we get

$$
|u-\mathscr{U}| \leq M e^{-l t} \text { in } E^{+} .
$$

Since $M$ is independent of the choice of $T$, the result is true for all $t>0$ and this completes the proof of Theorem 3.1.

4. Numerical results. In this section, we describe the results of some numerical experiments for the problem (1.1), (1.2). We consider three physically interesting cases.

Case 1: $D(u)=u, K(u)=u^{2} / 2$.

Case 2: $D(u)=2 u, K(u)=u^{3}$.

Case 3: $D(u)=2 u, K(u)=u^{4}$.

We adopt the method of finite differences to obtain the solution of (1.1), (1.2). The travelling wave solution is obtained by solving (2.3) numerically subject to the condition (2.4).

In order to approximate the solution of (1.1), (1.2), we construct a difference approximation on a mesh with uniform spacing $h$ in the $x$ direction and $k$ in the 
$t$ direction. We then solve the problem in the rectangle $O A B C$, where $O A=m h$ and $O B=n k$, where $m$ and $n$ are positive integers. We enlarge the rectangle as we continue the integration. The method employed is implicit and forward in time. When we evaluate the solution at time level $t=j k$, where $j$ is any integer, the nonlinear terms in the equation and the coefficients $D(u), D^{\prime}(u)$, and $K^{\prime}(u)$ are evaluated at the previous time level $t=(j-1) k$. The solution involves inverting a tridiagonal matrix for each time level. For this purpose, we adopt LU factorization. After we move forward a sufficient number of steps in time, the approximate solution of the partial differential equation and the travelling wave solution get close to each other.

For all three cases, we have taken $u_{1}=0.9, u_{0}=0.1, h=0.01$, and $k=0.01$. The initial profile $u_{0}(x)$ is chosen so as to satisfy the conditions mentioned in (2.8). In Case 1, we have taken $u_{0}(x)=0.1+(1.6 /[1+\exp (10 x)])$. In Table 1 (see p. 636), we present results for Case 1 for a certain number of time steps, starting from $t=26$. The column marked "Max Diff" represents $\max _{x}[\tilde{u}(x, t)-\tilde{\mathscr{U}}(x, t) \mid$, where $\tilde{u}(x, t)$ and $\tilde{\mathscr{U}}(x, t)$ denote the numerical solution and the travelling wave solution, respectively. For Case $1, l \approx 0.01$ (one may recall $l$ from the proof of Theorem 3.1). The third column in Table 1 denotes [MaxDiff] $\exp (0.01 t)$. In Fig. 1 (see p. 638 ), we show the graphs of numerical solutions and the travelling wave for $t=26$ and $t=39$. It is clear that they get closer as $t$ increases. Similar results for Cases 2 and 3 are presented in Tables 2 and 3 (see p. 637) and Figs. 2 and 3 (see p. 639), respectively.

5. Conclusions. Theorem 3.1 states that the solution of the problem (1.1), (1.2) gets close to the travelling wave solution as $t$ increases in accordance with (3.1). This is clearly seen from numerical results in Figs. 1, 2, and 3 for specific choices of $D(u)$ and $K(u)$. Tables 1,2 , and 3 show that the theoretical prediction of the approach of the solution of the problem (1.1), (1.2) to the travelling wave as given by Theorem 3.1 is quite accurate, again for the specific choices of $D(u)$ and $K(u)$. This study confirms the importance of the travelling wave solutions as intermediate asymptotics to more general initial-boundary value problems. 
TABLE 1. $D(u)=u, K(u)=u^{2} / 2$

\begin{tabular}{|c|c|c|}
\hline$t$ & Max Diff & [Max Diff] $\exp (0.01 t)$ \\
\hline 26. & 0.0143 & 0.0185 \\
\hline 27. & 0.0143 & 0.0187 \\
\hline 28. & 0.0141 & 0.0187 \\
\hline 29. & 0.0139 & 0.0185 \\
\hline 30. & 0.0135 & 0.0182 \\
\hline 31. & 0.0131 & 0.0179 \\
\hline 32. & 0.0127 & 0.0175 \\
\hline 33. & 0.0122 & 0.0170 \\
\hline 34. & 0.0117 & 0.0164 \\
\hline 35. & 0.0111 & 0.0158 \\
\hline 36. & 0.0106 & 0.0151 \\
\hline 37. & 0.0100 & 0.0145 \\
\hline 38. & 0.0094 & 0.0138 \\
\hline 39. & 0.0094 & 0.0139 \\
\hline
\end{tabular}


TABLE 2. $D(u)=2 u, K(u)=u^{3}$

\begin{tabular}{|c|c|c|}
\hline$t$ & Max Diff & [Max Diff] $\exp (0.001 t)$ \\
\hline 40. & 0.1246 & 0.1296 \\
\hline 41. & 0.1120 & 0.1167 \\
\hline 42. & 0.0995 & 0.1037 \\
\hline 43. & 0.0869 & 0.0907 \\
\hline 44. & 0.0744 & 0.0778 \\
\hline 45. & 0.0621 & 0.0650 \\
\hline 46. & 0.0499 & 0.0523 \\
\hline 47. & 0.0383 & 0.0401 \\
\hline 48. & 0.0277 & 0.0290 \\
\hline 49. & 0.0189 & 0.0198 \\
\hline
\end{tabular}

TABLE 3. $D(u)=2 u, K(u)=u^{4}$

\begin{tabular}{|c|c|c|}
\hline$t$ & Max Diff & [Max Diff] $\exp (0.001 t)$ \\
\hline 40. & 0.1203 & 0.1253 \\
\hline 41. & 0.1085 & 0.1130 \\
\hline 42. & 0.0967 & 0.1008 \\
\hline 43. & 0.0850 & 0.0887 \\
\hline 44. & 0.0735 & 0.0768 \\
\hline 45. & 0.0389 & 0.0407 \\
\hline 46. & 0.0294 & 0.0308 \\
\hline 47. & 0.0214 & 0.0224 \\
\hline 48. & 0.0158 & 0.0166 \\
\hline
\end{tabular}




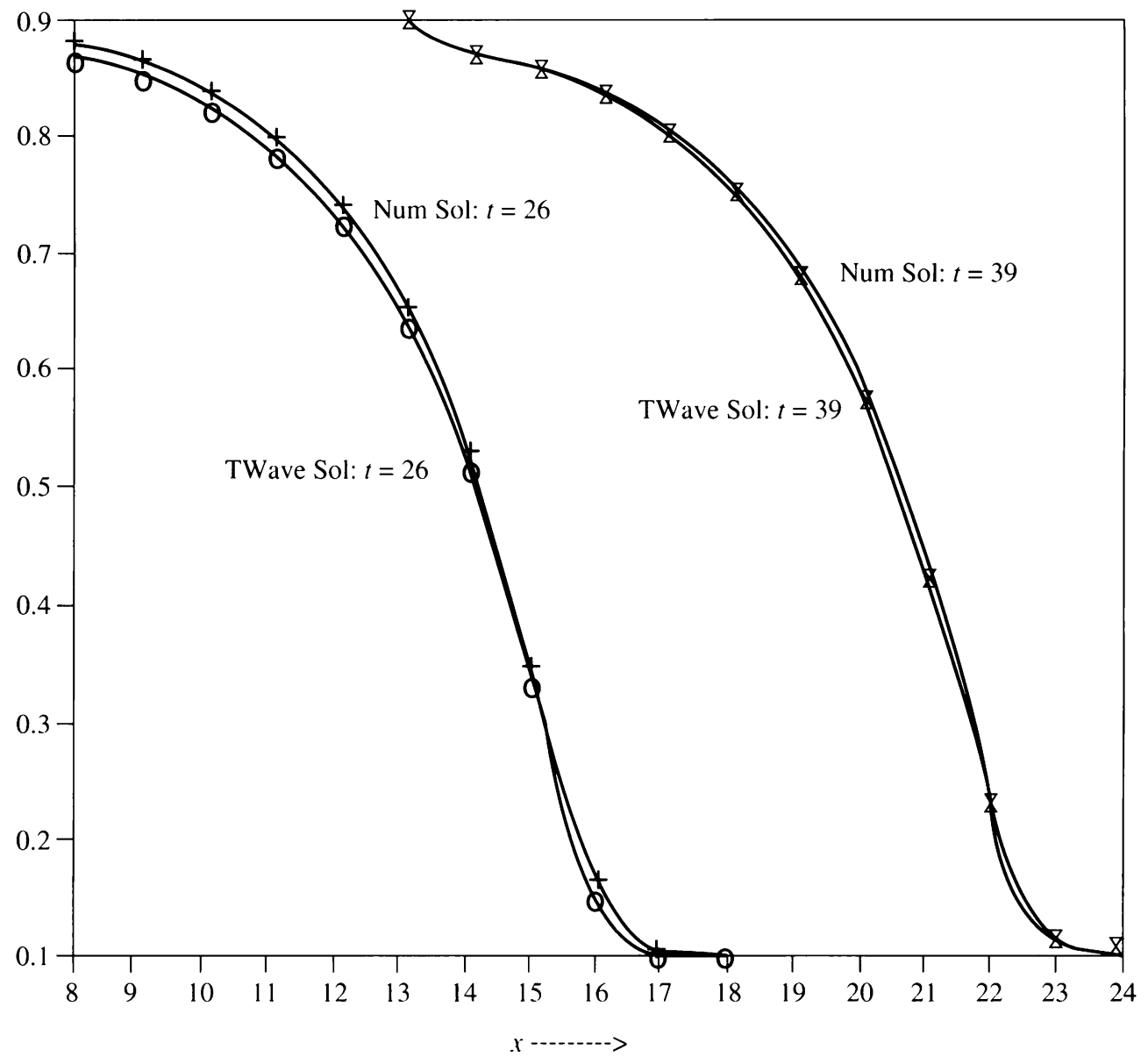

FIG. 1. $D(u)=u, K(u)=u^{2} / 2$ 


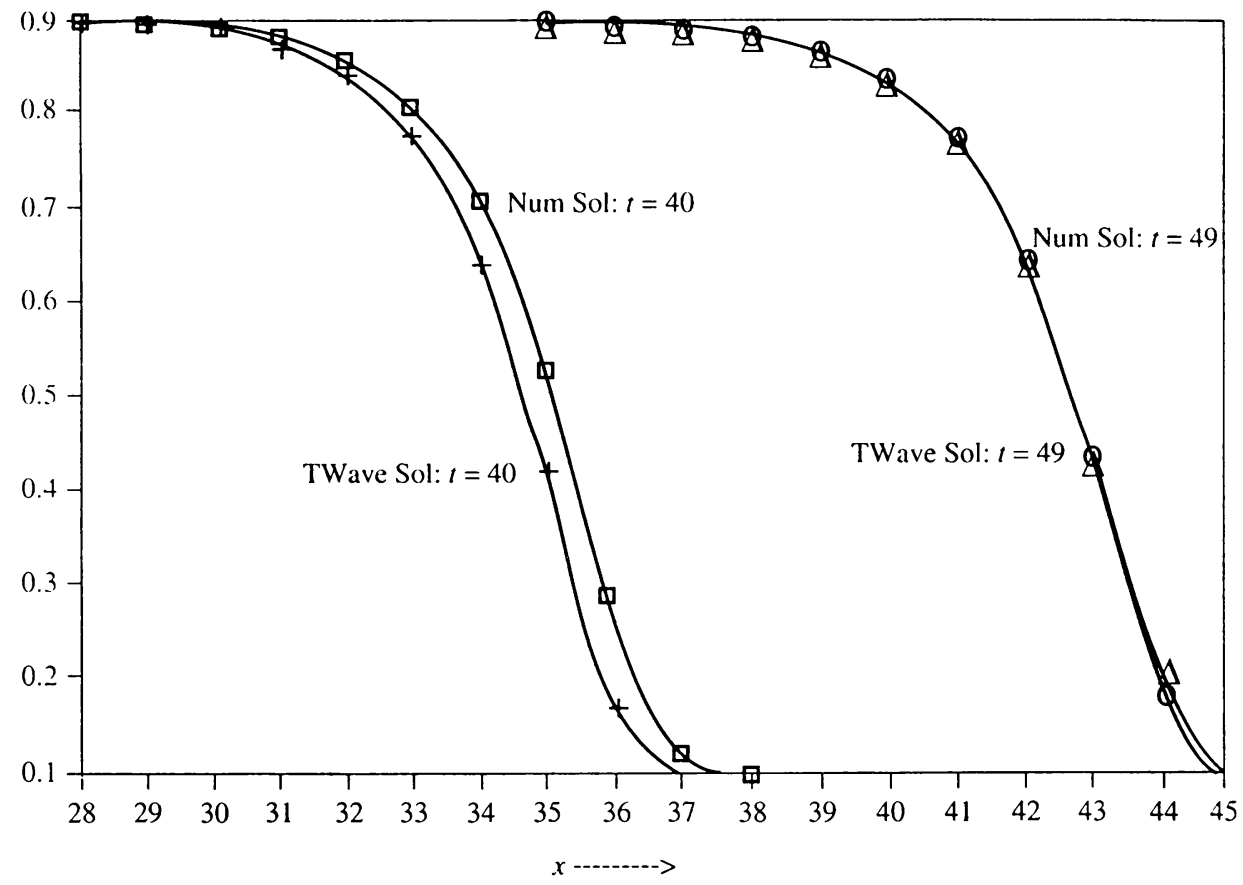

FIG. 2. $D(u)=2 u, K(u)=u^{3}$

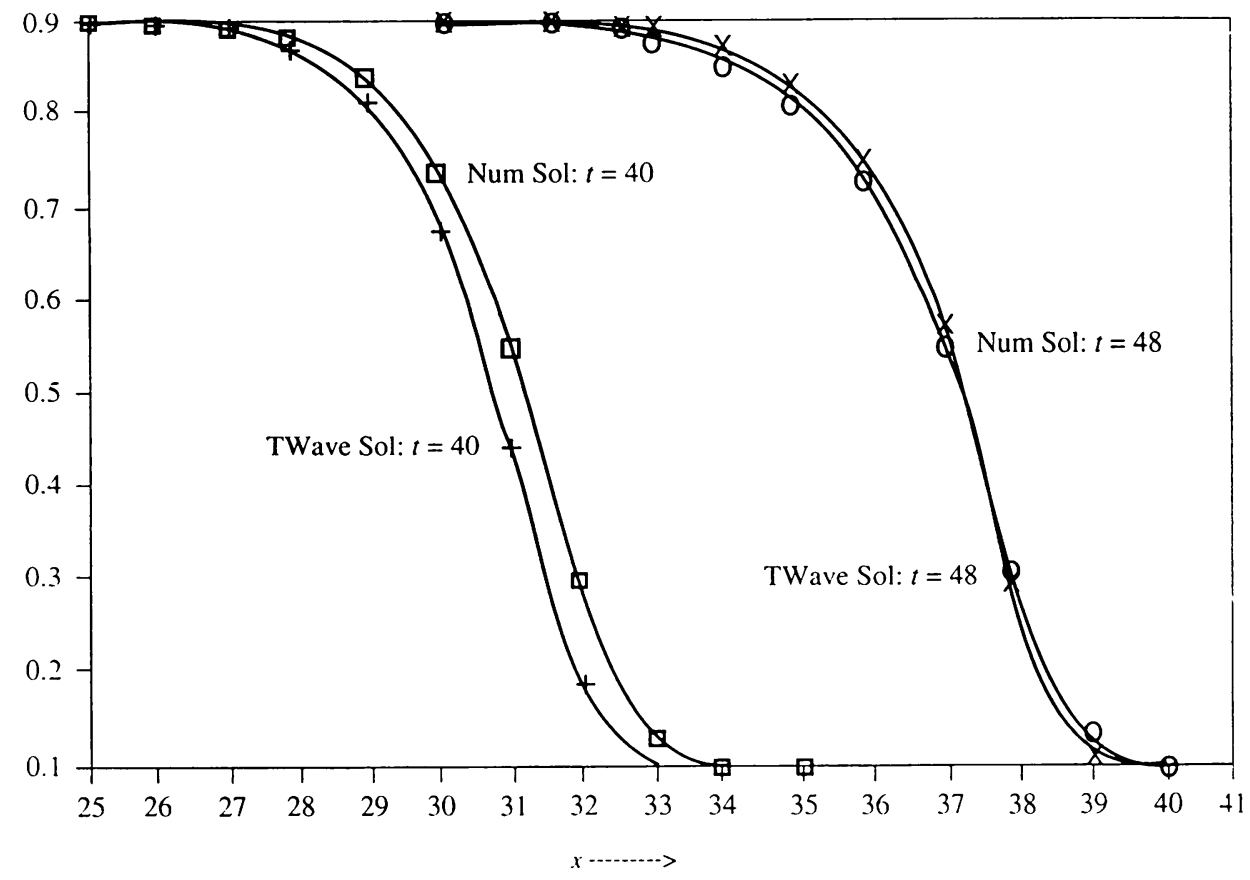

FIG. 3. $D(u)=2 u, K(u)=u^{4}$ 


\section{REFERENCES}

[1] G. I. Barenblatt and Y. B. Zel'dovich, Self-similar solutions as intermediate asymptotics, Ann. Rev. Fluid Mech. 4, 285-312 (1972)

[2] J. Bear, Dynamics of Fluids in Porous Media, Elsevier, New York, 1972

[3] R. H. Brooks and A. T. Corey, Properties of porous media affecting fluid flow, J. Irrigation Drainage Div. ASCE 92, 61-88 (1966)

[4] J. Canosa, Diffusion in nonlinear multiplicative media, J. Math. Phys. 10, 1862-1868 (1969)

[5] J. Canosa, On a nonlinear diffusion equation describing population growth, IBM J. Res. Develop. 17, 307-313 (1973)

[6] B. H. Gilding and L. A. Peletier, The Cauchy problem for an equation in the theory of infiltration, Arch. Rational Mech. Anal. 6, 127-140 (1976)

[7] R. E. Grundy, Asymptotic solution of a model non-linear convective diffusion equation, IMA J. Appl. Math. 31, 121-137 (1983)

[8] S. Irmay, On the hydraulic conductivity of unsaturated soils, Trans. Amer. Geophys. Union 35, 463-467 (1954)

[9] N. V. Khusnytdinova, The limiting moisture profile during infiltration in to a homogeneous soil, Prikl. Mat. Mekh. 31, no. 4, 770-776 (1967)

[10] M. Krzyzanski, Bull. Acad. Polon. Sci. Ser. Sci. Math. Astronom. Phys. 7, 131-135 (1959)

[11] L. A. Peletier, Asymptotic behaviour of temperature profiles of a class of non-linear heat conduction problems, Quart. J. Mech. Appl. Math. 23, 441-447 (1970)

[12] J. R. Philip, Flow in porous media, Ann. Rev. Fluid Mech. 2, 177-204 (1970)

[13] P. L. Sachdev, Nonlinear Diffusive Waves, Cambridge Univ. Press, 1987

[14] J. F. Scott, The longtime asymptotics of solution to the generalized Burgers equation, Proc. Roy. Soc. London Ser. A 373, 443-456 (1981)

[15] J. Serrin, Asymptotic behaviour of velocity profiles in the Prandtl boundary layer theory, Proc. Roy. Soc. London. Ser. A 299, 491-507 (1967)

[16] D. Swartzendruber, The flow of water in unsaturated soils, Flow Through Porous Media (R. J. M. Dewiest, ed.), Academic Press, New York; 1969, pp. 215-292

[17] C. J. Van Duyn and L. A. Peletier, Asymptotic behaviour of solutions of a non-linear diffusion equation, Arch. Rational Mech. Anal. 65, 363-377 (1977)

[18] C. J. Van Duyn and L. A. Peletier, A class of similarity solutions of the nonlinear diffusion equation, Nonlinear Anal., Theory, Methods Appl. 1, 223-233 (1977) 